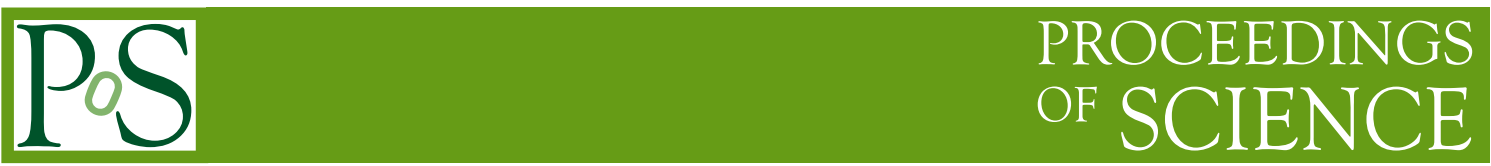

\title{
Upgrade of the ATLAS Muon Barrel Trigger for HL-LHC
}

\author{
Silvia Biondi* on behalf of the ATLAS Collaboration \\ University and INFN, Bologna (IT) \\ E-mail: silvia.biondi@cern.ch
}

\begin{abstract}
The current ATLAS muon trigger in the barrel region (in the pseudo-rapidity range $|\eta|<1.05$ ) is based on three layers of Resistive Plate Chambers detectors. It was designed to run for 10 years at the LHC luminosity of $10^{34} \mathrm{~cm}^{-2} \mathrm{~s}^{-1}$ and operated successfully and with high selectivity during the first period of the LHC. In order to ensure a stable performance of the RPCs until 2035 at the higher rates and at luminosities of $5-7 \times 10^{34} \mathrm{~cm}^{-2} \mathrm{~s}^{-1}$ expected at HL-LHC, the chambers will have to be operated with reduced operating voltage and gas gain to respect the original design to limit the aging of the detector. The ATLAS muon collaboration proposes an upgrade of the system by installing an inner layer of new generation RPCs during the LHC shutdown foreseen for the year 2023. This new layer will increase the system redundancy and therefore allow operation with high efficiency and high selectivity during the HL-LHC phase. The insertion of this new layer will also increase the geometrical acceptance in the barrel region from $75 \%$ to $95 \%$. Moreover, the additional measurements provided by the inner layer will improve the resolution on the reconstructed muon momentum. The trigger electronics will be upgraded with a more flexible system capable to operate at the expected level- 0 rate of $1 \mathrm{MHz}$. The first $10 \%$ of the system, corresponding to the edges of the inner barrel even sectors, has been already approved by ATLAS and will be installed in 2018, to reinforce the trigger in the region between barrel and endcap.
\end{abstract}

The European Physical Society Conference on High Energy Physics

22-29 July 2015

Vienna, Austria

* Speaker. 


\section{Introduction}

The architecture of the present trigger system in the ATLAS Muon Barrel was designed according to a reference luminosity of $10^{34} \mathrm{~cm}^{-2} \mathrm{~s}^{-1}$ with a safety factor of 5 , with respect to the simulated background rates, now confirmed by LHC Run-1 data. HL-LHC [1] will provide a luminosity 5 to 7 times higher, and a consequent higher background. The schedule for the upgrades is shown in figure 1.

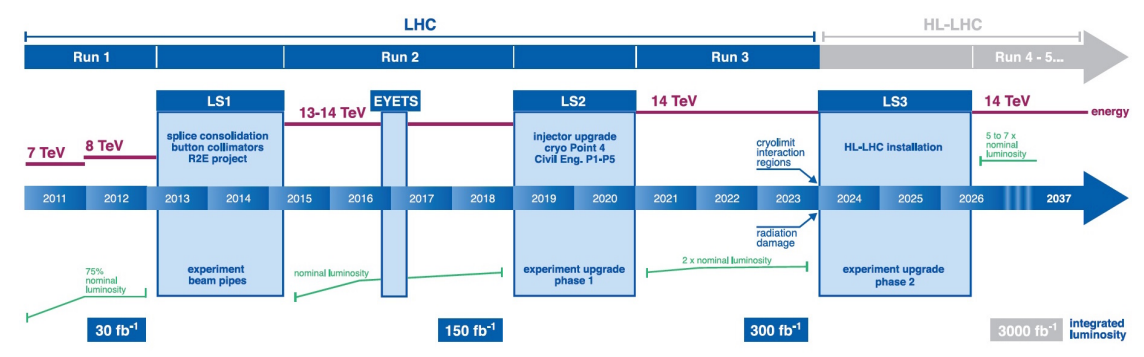

Figure 1: High Luminosity LHC time schedule [1].

The main reasons of the proposed upgrade are the aging effects of the detector caused by the increase of the rates and background conditions and the current muon trigger acceptance in the barrel region, which is above $73 \%$, due to the presence of the toroid magnets supports and ATLAS services. The ATLAS muon collaboration has proposed an upgrade plan, both for the detectors and the trigger readout electronics, which will ensure the required performance for the next twenty years of LHC operation. This consists in installing a layer of new generation RPCs in the inner barrel, to increase the redundancy and the background rejection, and to provide almost full acceptance. The first $10 \%$ of the system, corresponding to the edges of the inner barrel even sectors (BIS), has been already approved by ATLAS and will be installed in 2018, for the Phase-I (2019 2020), to reinforce the trigger in the region between barrel and endcap. To match the performance requirements (higher hit rate capability), the new smaller gap RPCs will have a different structure and materials, and high performance front-end electronics. We will illustrate the performance of the new detectors and trigger system, as well as the impact on the ATLAS physics performance.

\section{The ATLAS muon spectrometer}

The ATLAS detector [2] is a multi-purpose apparatus with a cylindrical geometry covering almost the entire solid angle around the interaction point.

The muon spectrometer, situated in the outer region of ATLAS, is built with four different technologies: Monitored Drift Tubes (MDT) and and Cathode Strip Chambers (CSC) measure with high precision the bending coordinate up to $|\eta|<2.7$, Resistive Plate Chambers (RPC) in the barrel region $(|\eta|<1.05)$ and Thin Gap Chambers (TGC) in the endcap regions $(1.05<|\eta|<2.4)$ also provide the non-bending coordinate $\phi$ and the trigger signal. The ATLAS trigger system is based on a hardware based level (L1) and a software based higher-level trigger (HLT). It reduces the rate from $40 \mathrm{MHz}$ to about $200 \mathrm{~Hz}$. The Level 1 Muon Trigger is based on RPC and TGC hits and defines the Regions of Interest (ROI) that are used to seed the HLT. 
In the barrel region, three layers of trigger chambers are implemented and the schematic layout of the trigger system is shown in figure 2. Two layers (RPC1 and RPC2) sandwich the MDTs of the middle layer, while the third one (RPC3) is located close to the outer MDT layer. The tracks of the muons are bent in the toroidal field and the deviation from a straight line is inversely proportional to the momentum of the muons. This deviation is measured considering the slope of the track segment between two trigger chambers from the slope of a straight line between the interaction point and the hit in a reference layer called the pivot plane, which is the second layer in the barrel (RPC2). For the low (high)- $p_{\mathrm{T}}$ trigger in the barrel, for example, the slope between RPC2 and $\mathrm{RPC} 1$ (RPC3) is compared to the slope between the interaction point and RPC2.

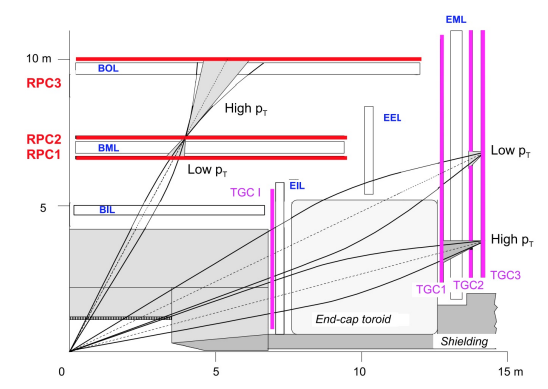

Figure 2: The Muon trigger scheme in the barrel region [2].

\section{Upgrade motivations}

\subsection{RPC detector coverage}

The actual RPC detector geometrical coverage is limited to $73 \%$ because of the ATLAS mecchanical support structure. The RPC trigger efficiency for muons with $p_{\mathrm{T}}>10 \mathrm{GeV}$ is shown in figure $3 \mathrm{a}$, versus the azimuthal and polar angles. The orange and red regions correspond to lower trigger efficiencies, due to the reduced RPC detector acceptance in the regions where there are toroid mechanical supports. The regions where there are no RPC detectors at all are marked as white. In figure 3b, L1 muon trigger efficiency is studied as a function of $\eta$, using both real and simulated muons (2012 data and MC, respectively). Two different trigger requirements are considered: MU10 is the trigger for muons with $p_{\mathrm{T}}$ above approximately $10 \mathrm{GeV}$ (required coincidence in only two stations in RPC) and MU11 is the trigger for muons with $p_{\mathrm{T}}$ above approximately 11 $\mathrm{GeV}$ (this threshold uses the same coincidence windows as MU10, except now requiring threestation coincidence in the RPC). The main inefficiencies are in the BM (Barrel Medium) region, because of the toroid ribs in small sectors that contain the coils, and in the feet region, because of the toroid feet structure. The feet region coverage has been recovered $(\sim 4 \%)$ in Run-2 using additional RPC chambers installed outside the feet structure. One possibility to increase the current detector coverage is to insert additional RPC chambers in the BI (Barrel Inner) region, where there are no mechanical structures and services.

\subsection{RPC trigger rates and extrapolations}

Several studies are made to estimate the RPC trigger rates in the Phase- 2 conditions and to test the RPCs aging issue. 


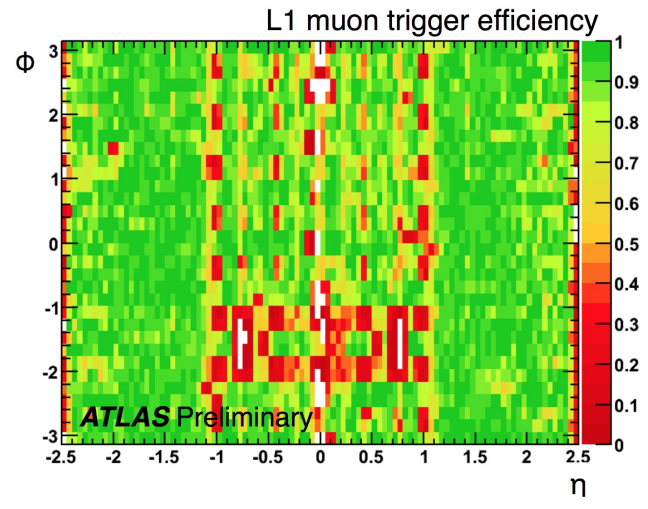

(a)

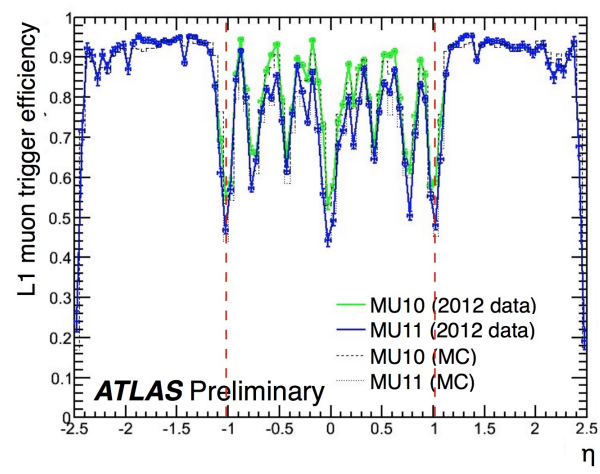

(b)

Figure 3: RPC trigger efficiency: a) efficiency map of the L1 muon trigger for muons with $p_{\mathrm{T}}>10$ $\mathrm{GeV}$ and b) efficiency as a function of $\eta$ for different trigger requirements [5].

The figure 4a shows the L1 MU20 Barrel muon rates (trigger for muons with $p_{\mathrm{T}}$ above approximately $20 \mathrm{GeV}$ ) as a function of the instantaneous luminosity, using 2012 data. Fake triggers are mainly due to secondary particles, like protons produced in dense materials such as the magnets. The figure $4 \mathrm{~b}$ [7] shows, for each region of the middle layer of the barrel spectrometer, the background rate measured in 2012 at a luminosity of $0.6 \times 10^{34} \mathrm{~cm}^{-2} \mathrm{~s}^{-1}$, extrapolated to $1.0 \times 10^{34}$ $\mathrm{cm}^{-2} \mathrm{~s}^{-1}$ at a center of mass energy of $14 \mathrm{TeV}$. At large $\eta$ the rates are above the expectations and will certainly exceed the $100 \mathrm{~Hz} / \mathrm{cm}^{2}$ during HL-LHC. The maximum value foreseen for HL-LHC is not sustainable for the detector, which has been realised for a much lower luminosity. Extrapolating 2012 data to Phase-II luminosity give a trigger rate of $\sim 150 \mathrm{~Hz} / \mathrm{cm}^{2}$ in average and of $\sim 300$ $\mathrm{Hz} / \mathrm{cm}^{2}$ in the sectors where the environment is busier.

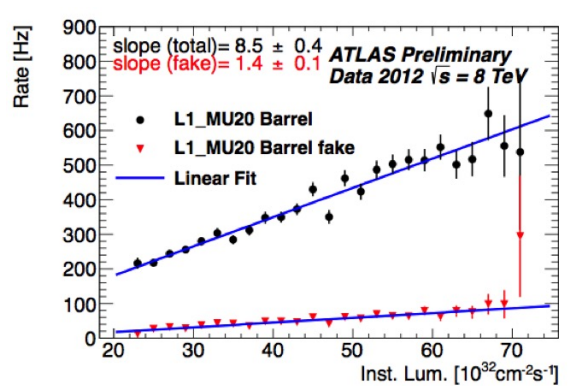

(a)

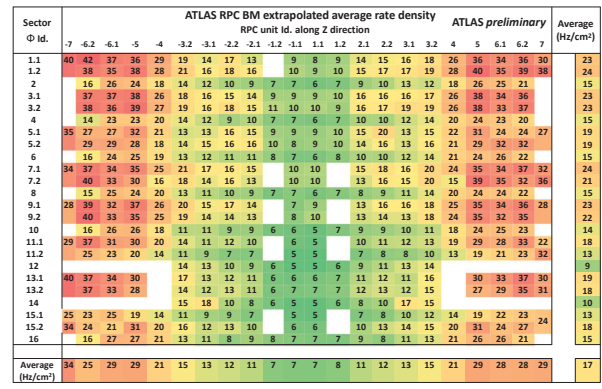

(b)

Figure 4: RPC trigger rates extrapolations with 2012 data and simulating Phase-2 conditions: a) L1 MU20 Barrel rates, 2012 data [4] and b) RPC middle layer background map measured at a luminosity of $0.6 \times 10^{34} \mathrm{~cm}^{-2} \mathrm{~s}^{-1}$ and extrapolated to $1.0 \times 10^{34} \mathrm{~cm}^{-2} \mathrm{~s}^{-1}$ at a center of mass energy of $14 \mathrm{TeV}$ [3].

\section{RPC upgrade proposal for Phase-I}

During Run-3, the expected total rate of the Level-1 single muon trigger (with transverse 
momentum $p_{\mathrm{T}}>20 \mathrm{GeV}$ ) will rise up to $57.6 \mathrm{kHz}$, if no measures are taken, while ATLAS can allocate only $25 \mathrm{kHz}$ for muon triggers out of a total Level-1 bandwidth of $100 \mathrm{kHz}$ [6].

The rate can be reduced requiring a coincidence between the End Cap trigger and the passage through an inner plane. The Barrel is divided in 16 sectors in azimuthal angle $\phi$, divided into small and large sectors. The small sectors contain the coils of the Barrel toroidal magnetic field. For the large sectors of the spectrometer this inner plane is covered by the TGCs in the inner layer of the End Cap (EI), while in the small sectors the EI chambers do not extend in the transition region. The proposal is to add RPCs to BIS 7-8 chambers, which are the inner Barrel MDT chambers that cover the transition region, as shown in figure 5.

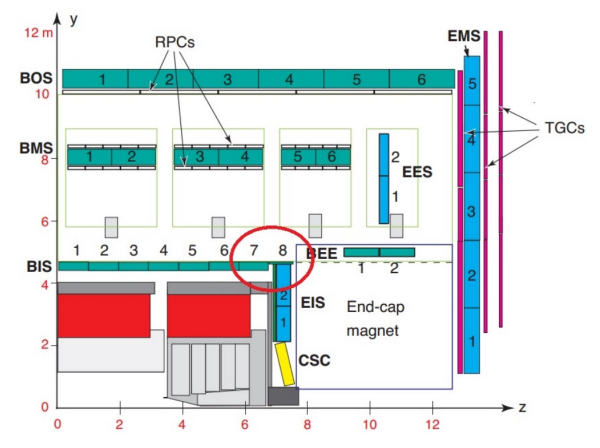

Figure 5: Scheme of the small sectors of the Barrel of the ATLAS muon spectrometer. The red circle highlights the BIS7-8 chambers, where the RPCs have to be added [6].

\section{RPC inner layer upgrade proposal for Phase-II}

The insertion of a new RPC inner layer (triplet or quadruplet) could allow to increase the current detector coverage up to $96 \%$. The proposal is to have 4 concentric RPC chambers (composed by more than one layer each), that means a group of 10 layers. The result increased redundancy allows to cope with the HL-LHC foreseen RPC hit rates.

In this condition, the BM station can be used with a 2/4 majority (that means a 2 out of 4 chambers coincidence) instead of the $3 / 4$ (a 3 out of 4 chambers coincidence), allowing a consistent reduction of gap efficiency without affecting the trigger efficiency. For most of the chambers, the new operating point restores a reasonable safety factor. In this new configuration, the trigger robustness is increased as well and the increased lever arm allows for a sharper momentum threshold.

\subsection{RPC study strategy}

The RPC study concerns the comparison between different RPC requirements. The comparison between old and new configuration is possible by including the L0 trigger, the so-called RPC0. This is simulated with the MDT chambers in the BI (Barrel Inner) region.

The analysis strategy consists in the simulation of the trigger algorithm to simulate different possible triggers, the reconstruction of a candidate muon hits for each event (like a pattern recognition), the association between RPC hits and MDT segments with a fixed $\Delta \eta$ and, at the end, the estimation of the acceptances using both the RPC info (obtained with the trigger simulation) and the MDT segments info. 


\section{Conclusions}

The studies shown in this document strongly validate the ATLAS muon collaboration proposal of an appropriate upgrade plan, involving both detector and trigger-readout electronics, to guarantee the performance required by the physics program for the 20 years scheduled. This consists in installing a layer of new generation RPCs in the inner barrel, to increase the redundancy and the background rejection, and to provide almost full acceptance.

This study, also, demonstrates the necessity of an additional RPC layer and the improvement in the acceptance. In fact, as shown in figures $6 \mathrm{a}, 6 \mathrm{~b}$ and $6 \mathrm{c}$, the acceptance improves from $73 \%$ to $96 \%$, adding the so-called RPC0, which is, in this study, simulated with the MDT chambers in the BI region.

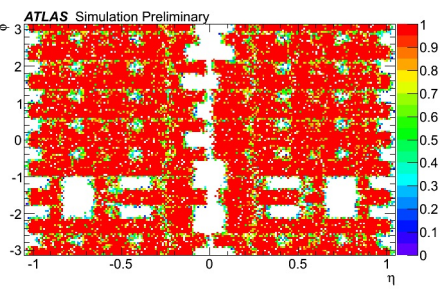

(a)

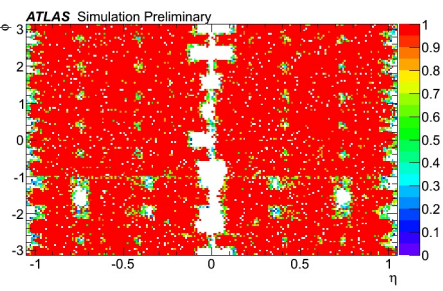

(b)

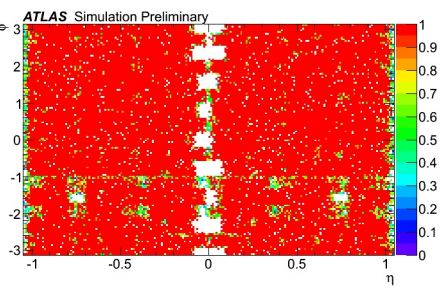

(c)

Figure 6: Trigger acceptance for different RPC trigger requirements, using all current RPCs and the simulated RPC0: a) current trigger requires hits on RPC1, RPC2 and RPC3, b) the trigger requirement is any 3 out of 4 RPCs and c) the trigger requirement is (any 3 out of 4) OR inner layer RPC0 [3].

\section{References}

[1] http://hilumilhc.web.cern.ch/about/hl-lhc-project

[2] ATLAS Collaboration, The ATLAS Experiment at the CERN Large Hadron Collider, 2008 JINST 3 S08003.

[3] ATLAS Muon Public Results web page, https://twiki.cern.ch/twiki/bin/view/AtlasPublic/MuonPublicResults

[4] ATLAS Muon Trigger Public Results web page, https://twiki.cern.ch/twiki/bin/view/AtlasPublic/MuonTriggerPublicResults

[5] L1 Barrel Muon Trigger Efficiency with 2012 Data, https://cds.cern.ch/record/1647612

[6] The ATLAS Collaboration, Technical Design Report for the Phase-I Upgrade of the ATLAS TDAQ System, CERN-LHCC-2013-018, ATLAS-TDR-023

[7] G. Aielli, High Luminosity (HL)LHC perspectives for the ATLAS RPC system, RPC 2014, Tsinghua, Beijing 2014 